\title{
THE ALLOCATION OF FIRE STATIONS USING SET COVER APPROACHES
}

\author{
Nik Fatihah Nik Lah ${ }^{1}$, Rosshairy Abd. Rahman ${ }^{2}$ \\ ${ }^{1,2}$ Decision Science Department, School of Quantitative Sciences, Universiti Utara Malaysia, \\ 06010 Sintok Kedah, MALAYSIA \\ *Corresponding E-mail: shairy@uum.edu.my
}

\begin{abstract}
Fireman is one of the most significant job in our country with multiple important task especially during fire and accident. Thus, the allocation of sufficient fire station is vital to cover the whole community within specific time. Consequently, this paper aims to build a framework to determine the specific number of fire station to be allocated in order to serve the whole community in Changlun Town, Kedah using set cover approaches. This approach is regularly employ in allocating the minimum number of fire station to cover the specific area with specific time limit. This study uses one of the core model in set cover problem which is known as Location Set Cover Problem. This model is designed for determining the specific number of fire station to ensure that all area in Changlun are covered within 15 minutes time. Greedy heuristic is used as a technique to accomplish the objective of this paper and gives out the best solution by proposing a specific location of these fire stations. This solution can be a guidance to government in designing and developing Changlun in the future.
\end{abstract}

Keywords: Set Cover, Location Set Cover Problem, Greedy Heuristics

DOI: https://doi.org/10.30880/jtmb.2018.05.03.002

Received: January 16, 2018

Accepted: January 25, 2018

Published: December 15, 2018

\subsection{Introduction}

The location of emergency service stations (ESS) like fire stations, hospitals, and police stations is vital in achieving an effective and reliable emergency response system. Accidents, fires, illness, and natural disaster are some examples that will cause the fatalities and disabilities, thus they may be reduced through an effective planning of the locations of their stations. As time passes by the communities will become larger and demographic also changes, thus it become essential to replace the existing ESS or add more stations therefore the increasing demands for emergency response from public can be fulfilled.

The location of fire stations plays a significant role since the incident may occur any time at any place, thus the time taken for fire brigades to arrive is a crucial part as life safety of the public is one of firemen's main roles. Firemen help to overcome the loss of life from fires, deliver first aid during medical emergencies, and rescue people from hazardous and risky situation such as crashed vehicles and overturned vehicles. Other than that, firemen act as an incident stabilization which indicates that several incidents can potentially inflate if not quickly recovered like hazardous material releases, floods, forest fires, and attacks from terrorist which could cause severe damage. According to these situations, firemen need to work quickly, strategically, and systematically in order to minimize the potential risk of danger as well as the level of damage caused by these incidents.

The nature of fireman requires to arrive at a specific destination and provide community service as fast as possible or within a time limit, therefore the damage of property and the risk of 
life are reduced. The fire that is left unhandled for a longer time will spread more, thus the people who are trapped in the incident will probably have the less chance to survive. Seeing this situation, it is significant to explore the specific locations of fire stations that will guarantee fast operating pattern which is the emergency vehicles to be able to respond as fast as possible, or within a defined time limit.

The strategic location of fire station is important as the location and total number of fire station have given a big impact to the whole society. In addition, the number of fire station to be built should be determined with minimum budget. This indicates that there is a need to determine the distribution of strategic locations within the minimum budget as to ensure the effectiveness of fire protection and time distance. It means the best locations that assure the lowest possible response times made with minimum budget need to be discovered. A set cover approach is to be used in order to determine the exact number of fire station in Changlun town. This problem is suitable to solve using set cover approach that able to find the exact number of fire station to be placed so that can give a full coverage to community members in Changlun Town.

Set covering problem is one of the most significant discrete optimization problem because it represents as a model in real world problems and it is regarded as a classical problem in computer science and theory of complexity. Some examples of real world problems that can be modelled as set covering problem are nurse scheduling problem, airline crew scheduling, vehicle routing, resource allocation, and facility location problem which is the main aim of this work. Set covering problem is defined as a problem of an m-row column and n-row column that is to be covered with a subset of columns at a minimal cost (Beasley \& Chu, 1996). There are many models have been formulated and applied on problems related to the facility location and abundance of literature have been discovered.

The aim of this paper therefore is to use a set covering model in selecting the lowest number of fire stations and at the same time could serve all locations in such a way that every single location will have equal benefits in terms of emergency services from the fire stations.

The objective of this study is to find the minimum number of fire stations to be built and the best place to locate the fire stations for handling fire incidents within 15 minutes response time.

\subsection{Literature Review}

Emergency Service Station (ESS) location problem has become the centre of attraction for many researchers and abundance of articles existed regarding on this topic, mainly narrowing and focusing on locating emergency medical stations (EMS) and ambulances. Although the two problems are similar, fire stations may involve special specifications such as types of varied vehicles and equipment, costs of relocation and location, and the risk factors associated with the points of demand. There has been a long tradition of research within the field of determining optimal locations of ESS, with the first introduction to the field being the Location Set Covering Problem (LSCP) (Toregas et al., 1971). Thus, many different kind of models have since then been discovered and formulating as well as testing different objectives and different constraints.

The formulation of LSCP has been related to three assumptions, firstly is that the demand points can be characterized as a finite number of points where the station can be located is available. This first assumption stated that the demand and supply points are simplified in a way that allows areas to be grouped into specific demand points, instead of using each single household or similar as a demand point. The second assumption is about the minimum distance or response time between any pair of demand-points and supply-points are already known. Then, the third assumption stated that all the demand points have the same chance of locations possibility for stations. However, LSCP model also has two additional assumptions including that this model 
has an unlimited resources and every facility is able to handle all calls within its coverage area. These two additional assumptions are not representation of the real world problem as the unlimited resources is surely impossible because we need to deal with a certain public budget and also a facility seems cannot be able to handle all calls since additional incidents might occur while it is busy handling an incident.

However, all the different models in finding the optimal locations of ESS have a common aim which is the models look to dig out the sites that should be used to locate the facilities of emergency service within, when a number of demand points and possible facility location sites are given. The objective of all models is to figure out the best method to allocate the available equipment of firemen, hence a required level of service can be optimized (Badri, Mortagy \& Alsayed, 1998). There is one assumption existed regarding all different models that they also have in common which is all the models simplify the problem by treating emergency calls generated from discrete demand points ( $\mathrm{Li}$ et al., 2011). The differences between the models are in term of the type of objective that the models try to optimize and the type of constraints impose on the model. For instance, LSCP aims to place the lowest number of facilities but able to cover all demand points at least once by a facility placed within a required response time, meanwhile the Maximum Covering Location Problem (MCLP) which is first introduced by (Church \& Revelle 1974) aims to serve as many population with a limited number of stations to be placed within the required response time.

The MCLP model ensures that population can be covered at maximize number within time standard with a limited number of facilities given, therefore the solution from this formulation model might not cover all demand points within the time standard but the model prioritize the demand point with a high number of people. Another two models discovered are Maximum Availability Location Problem (MALP) (Revelle \& Hogan, 1989) and Expected Covering Location Problem (MEXCLP) (Daskin, 1983) which are both defined as the models that aim to incorporate the busy fraction, thus determining the portion of a fraction of the available time the facilities are busy to handle calls for every day, as well as the reliability of facilities. The MALP model is extended formula from MCLP model, but this model adds a reliability level of alpha, therefore it aims to increase the population that has a facility available within the defined time limit at a reliability alpha level. MALP focuses on incorporation randomness into the model which means that the availability of the servers is different as the travel time is deterministic. Since the incidents occurred in the real life is not deterministic which is we unable to predict the time of incidents are going to happen, therefore the assumptions discussed in both LSCP and MCLP models that stated a server can handle all calls within its coverage area could be problematic. As a consequence, MALP model includes the probability of a server being available.

A significant assumption in the MALP model is the facility availability that defined as the ability of the facility to handle incoming calls and is independent of other facilities being in use. This kind of assumption is a simplification of the situation in real world, for example when a station has responded to a call but it requires some helps such as additional firemen or additional equipment in order to handle fire, thus the station might call another station to assist. There are two sub-separated formulas in MALP model that have been explored and introduced by (Revelle\& Hogan, 1989) which are known as MALP-I and MALP-II, thus both of the sub-models use different assumptions for the busy fraction. MALP-I sub-model assumes that all servers are having a global busy fraction and defines that the busy fraction is the same among all servers, whereas in MALP-II, a busy fraction is developed for each section of the city.

Another main model is the MEXCLP (Daskin, 1983) which is quite similar with the previous MALP model that focused on incorporating the possibility of stations are being busy, however MEXCLP model do not aim to fulfil a requirement of an availability of alpha. The model instead functions with a chance of service is being covered at every point and maximization the expected coverage for all demand points are pointed as its objective (Galvao, 
Chiyoshi \& Morabito 2005). The MEXCLP model is about the estimation of the expected number of demand that could be able to handle when dealing a limited budget within a defined time limit. Hence, this model denied the deterministic approach to ask for services and also against the assumptions in LSCP and MCLP models where all calls could be covered by a station. The crucial part in MEXCLP and MALP model that differs from each other is in term of objective function where in MEXCLP model, the objective function is increased by the additional stations since a lot of demand is expected to be handled, whereas in MALP model, the satisfaction of alpha reliability of availability is depends on sufficient stations in order to be counted in.

As a conclusion, LSCP, MCLP, MEXCLP, and MALP are considered as the core models in determining the optimal locations of ESS. It is cannot be denied that many other models have been discovered and explored since then but they are just the extended and modified version from these first four models in order to improvise the models for getting the better solution for a problem. In spite of that, the set covering problem is known as a hard problem and regarded as to be NP-complete since decades ago (Karp, 1972; Aho, Hopcroft \& UUman 1974), thus different approaches and methodologies might require to solve problem of locating fire stations. In view of this fact, the relative significance of heuristics to solve the set covering problem increases. Greedy Heuristic is one of the techniques to give a solution to this problem and this technique can be simply defined as a technique of making the locally optimal choice at each phase with the hope of finding a global optimum. In many kinds of problem, a greedy strategy does not promise an optimal solution, but nonetheless this strategy may yield locally optimal solutions which approximate a global optima solution within a reasonable time (Cormen, Leiserson \& Rivest 1990).

\subsection{Methodology}

The model that we considered for placing and opening the location of fire stations was the Location Set Covering Problem (Toregas et al., 1971). In the Location Set Covering Problem (Toregas et al., 1971) we are looking to figure out the number of fire stations that we will need to place, to be able to cover the whole area within a defined target response time. The area consists of 29 neighborhoods where the location of all neighborhood in Changlun are shown in Fig. 1 by searching through Google Maps. Meanwhile, Table 1 states the name of each neighborhood. This study is an initial research work; thus it is applied to a small-scale problem.

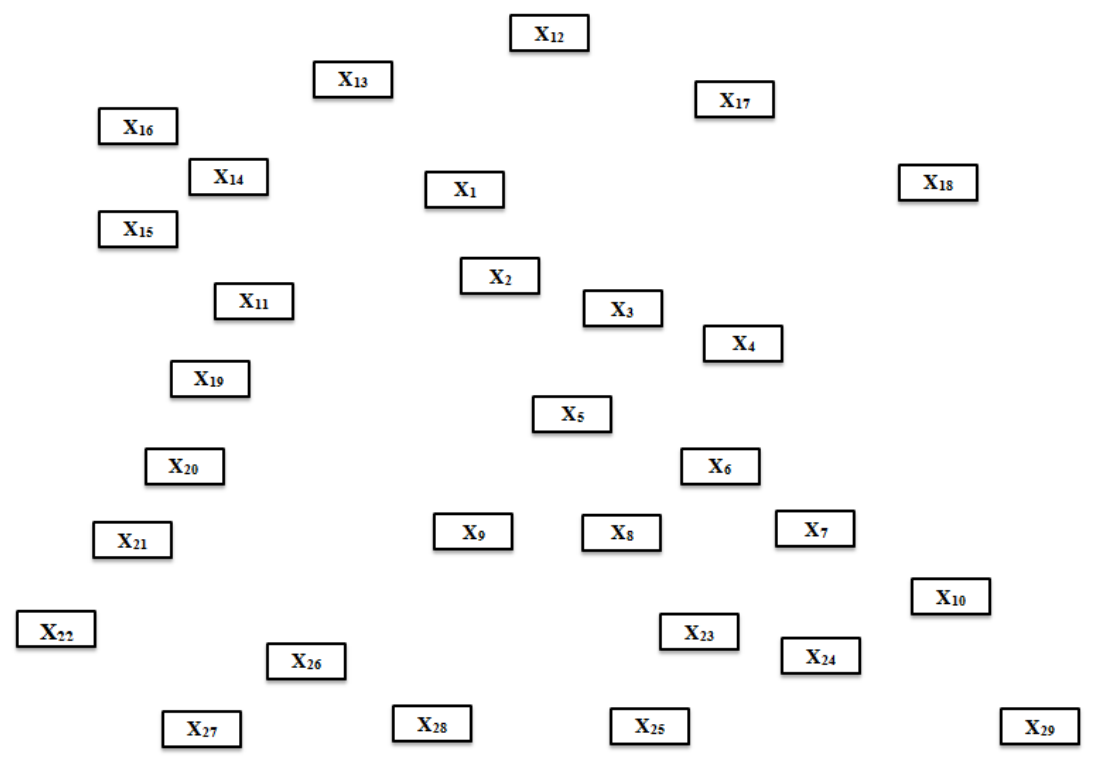

Figure 1: Location of all neighborhood in Changlun 
Table 1: List of all neighborhood in Changlun

\begin{tabular}{l|l|l|l}
\hline $\mathbf{X}_{\mathbf{i}}$ & Name & $\mathbf{X}_{\mathbf{i}}$ & Name \\
$\mathrm{X}_{1}$ & Kampung PayaNongmi & $\mathbf{X}_{16}$ & Kampung Sungai Atu \\
$\mathrm{X}_{2}$ & Kampung Batas & $\mathbf{X}_{17}$ & Kampung \\
$\mathrm{X}_{3}$ & Kampung Padang Halban & $\mathrm{X}_{18}$ & Kampung ChangkatSetol \\
$\mathrm{X}_{4}$ & Kampung ChangkatNibong & $\mathrm{X}_{19}$ & Kampung Baharu Che Harun \\
$\mathrm{X}_{5}$ & Kampung BendangPerang & $\mathrm{X}_{20}$ & Kampung Palas Baharu \\
$\mathrm{X}_{6}$ & Kampung Padang & $\mathrm{X}_{21}$ & Kampung Baharu Batu 4 \\
$\mathrm{X}_{7}$ & Kampung Darat & $\mathrm{X}_{22}$ & Kampung HutanPalas \\
$\mathrm{X}_{8}$ & Kampung Jeragan & $\mathrm{X}_{23}$ & Kampung Kubang \\
$\mathrm{X}_{9}$ & Kampung Lanterum & $\mathrm{X}_{24}$ & Kampung Kubang Betong \\
$\mathrm{X}_{10}$ & Kampung Kangar & $\mathrm{X}_{25}$ & Kampung Kubang Pasu \\
$\mathrm{X}_{11}$ & Kampung KepalaKerbau & $\mathrm{X}_{26}$ & Kampung Wang \\
$\mathrm{X}_{12}$ & Kampung TelagaDalam & $\mathrm{X}_{27}$ & Kampung Kubang Menerong \\
$\mathrm{X}_{13}$ & Kampung TitiKerbau & $\mathrm{X}_{28}$ & Kampung Baharu Batu 22 \\
$\mathrm{X}_{14}$ & Kampung Titi Ara & $\mathrm{X}_{29}$ & Kampung Wang Perah \\
$\mathrm{X}_{15}$ & Kampung LembahPalas & & \\
\hline
\end{tabular}

In this model, the most crucial parameter is the maximum response time or distance to any given demand point. The response time is defined as the time required from an alarm ringing at the fire station till the first truck to be arrived at the location of incident or demand point. Thus, the constraint considered in this model is every demand point should be encompassed within this response time which is 15 minutes (Jensen 2014). Hence, our main aim is to determine as few stations as possible, while ensuring that this service level can be reached. The problem is then solved using greedy set cover algorithm (Devanur, Mihali \&Vazirani 2004) and the steps are as follows:

Step 1:

If $\mathrm{C}_{\mathrm{i}}=0$, for any $\mathrm{i}, \mathrm{X}_{\mathrm{i}}=1$

Remove all constraints in which $X_{i}$ has a coefficient of 1 .

Step 2:

If $\mathrm{C}_{\mathrm{i}}>0$ for any $\mathrm{i}$, and

$X_{i}$ doesn't has a coefficient of 1 in any of the remaining constraints, then $X_{i}=0$.

Step 3:

For the remaining variables, compute $\mathrm{C}_{\mathrm{i}} / \mathrm{D}_{\mathrm{i}}$ where $\mathrm{D}_{\mathrm{i}} \mathrm{is}$ the number of constraints in which $\mathrm{X}_{\mathrm{i}}$ appears with a coefficient of 1 .

Choose the variable with the smallest $C_{i} / D_{i}$ and set $X_{i}=1$.

Remove all constraints in which $\mathrm{X}_{\mathrm{i}}$ has a coefficient of 1 .

Step 4:

If there are no more constraints, set all the remaining variables to 0 and stop. Otherwise go to Step 1. 


\subsection{Results and Discussions}

In this paper, we have considered the heuristic techniques in solving set covering problem. The problem consists of various models especially four core models i.e. LSCP, MCLP, MALP and MEXCLP. LSCP model has been chosen to locate fire stations since our aim is to give a full coverage to all neighborhood in Changlun without concerning much on a budget constraint. Greedy Heuristic technique is used in this case and we have obtained a near-optimal solution. The solution to this problem is that neighborhood $11\left(\mathrm{X}_{11}\right)$ and neighborhood $10\left(\mathrm{X}_{10}\right)$ are chosen to locate one fire station in each of these two neighborhoods. By locating one fire station in Kampung Kepala Kerbau $\left(\mathrm{X}_{11}\right)$ and another one fire station in Kampung Kangar $\left(\mathrm{X}_{10}\right)$, a full coverage of emergency services within 15 minutes to all neighborhoods in Changlun could be achieved.

\subsection{Conclusions}

Since set cover problem has various models including the four core models which are LSCP, MCLP, MALP and MEXCLP, thus these models could be used to compare which models could give highest benefit in real life problem especially when tight budget is considered. Furthermore, more constraint in bigger area will be considered in the future research. 


\section{References}

Aho, A. V., Hopcroft, J. E. \& UUman, J. D. (1974). The Design and Analysis of Computer Algorithms. Addison-Wesley, Reading.

Badri, M. A., Mortagy, A. K. \& Alsayed, C. A. (1998). A Multi-objective Model for Locating Fire Stations. European Journal of Operational Research, 110, 2, pp. 243.

Church, R. \& ReVelle, C. (1974). The Maximal Covering Location Problem. Regional Sciences Association, Papers-LA English, 32, pp. 101.

Cormen. Leiserson \& Rivest. (1990). Introduction to Algorithms. Chapter 17:Greedy Algorithms, pp. 329.

Daskin, M. S. (1983). A Maximum Expected Covering Location Model. Transportation Science, 17, 1, pp. 48. ReVelle, C. \& Hogan, K. (1989). The Maximum Availability Location Problem. Transportation Science, 23,3, pp. 192.

Devanur, N. R., Mihail, M. \& VVazirani, V. (2004). Strategy proof Cost-Sharing Mechanisms for Set Cover and Facility Location Games.

Jensen, S. (2014). Using Linear Programming Models to Optimize the Location of Fire Stations in Arhus.

Karp, R. M. (1972). Reducibility among Combinatorial Problems, In Complexity of Computer Computations (R E Miller \& J W Thatcher, eds), Plenum Press, New York.

Li, X., Zhao, Z., Zhu, X. \& Wyatt, T. (2011). Covering Models and Optimization Techniques for Emergency Response Facility Location and Planning: A Review. Mathematical Methods of Operations Research, 74, 3, pp. 281-310.

Toregas, C., Swain, R., ReVelle, C. \& Bergman, L. (1971). The Location of Emergency Service Facilities. Operations Research, 19, 6, pp. 1363. 